Accelerator Department

Assuciated Universities, Inc.

Upton, New York

INTERSI:CTING STORAGE ACCELERATOR NOTES

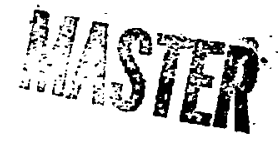

PREL IMINARY DESIGN OF LARGE ANGLE PROTON-PROTON ELASTIC

SCATTERING FOR ISABELLE

Jay Orear*

July 28, 1971

\title{
Theoretical Motivation
}

What is the energy dependence of large angle elastic scattering? At lower energies (up to $30-\mathrm{GeV}$ protons), do/dt goes as $\mathrm{s}^{-2}$ for fixed momentum transfer. Many theorists have speculated that $\mathrm{d} \sigma / \mathrm{dt}$ cannot drop faster than the fourth power of the e-p form factor. This happens to correspond to the measured $d \sigma / d t$ for $p \circ p$ at $30 \mathrm{GeV} / c$. Hence the prediction is that above $30 \mathrm{GeV}$ $\mathrm{d} \sigma / \mathrm{dt}$ is independent of energy. For example, at $-\mathrm{t}=7 \mathrm{Gev}^{2}, \mathrm{~d} \sigma / \mathrm{dt} \approx 10^{-33}$ $\mathrm{cm}^{2} / \mathrm{GeV}^{2}$ for $30-\mathrm{GeV}$ protons. An $\mathrm{s}^{-2}$ dependence would give a cross section 3000 times smaller. The predicted cross section is shown in Fig. 1. Another question concerns dips in the angular distribution. Is the dip at $t=1.4 \mathrm{GeV}^{2}$ still there, and will new dips appear at "infinite" energy?

\section{Experimental Layout}

Collinear crossing is used to maximize reaction rate and allow the scattered protons to clear the primary beams more easily. The preferred crossing option used the four dipoles in the order $(-++)$, i.e., polarities of the two upper half circles are the same.

Solid angle is 1 imited by the momentum transfer region corresponding to measurable cross sections. $d \sigma / d \omega$ for $p_{\perp}>5 \mathrm{GeV} / c$ will be too smail to give detectable rates. Hence $1<p_{\perp}<5 \mathrm{GeV} / \mathrm{c}$ is the region proposed. For $P_{\perp}=2.5+0.1 \mathrm{GeV} / \mathrm{c}$ a sol id angle $\Delta w=10^{-5} \mathrm{sr}$ is obtained. The four dipoles used to give beam crossing can also be used to deflect the scattered particles

\footnotetext{
* Laboratory of Nuclear Studies, Cornel1 University.
} 
away from the beams (see Fig. 2). Only secondaries of $p_{\perp}>1 \mathrm{GeV} / \mathrm{c}$ would reach the detectors shown in Fig. 2, hence the singles rates will be low enough. Parallel rays from the interaction region are focused to a point on the proportional chamber detectors. Each value of momentum transfer is at a different point on the detector.

\section{Count ing Rates}

We assume with bunching a luminosity $\mathcal{E}=5 \times 10^{33} \mathrm{~cm}^{-2} \mathrm{sec}^{-1}$. We assumo an interaction region $\ell=10 \mathrm{~m}$, and a solid angle $\Delta \omega=10^{-5} \mathrm{sr}$. At $p_{\perp}=2.5 \mathrm{GeV} / \mathrm{c}$ the $30-\mathrm{GeV}$ cross section is $\mathrm{d} \sigma / \mathrm{dt} \approx 1.7 \times 10^{-33} \mathrm{~cm}^{2} / \mathrm{GeV}^{2}$. The number of elastic scatters per second is then

$$
\begin{array}{rlrl}
N_{p p} & =\mathcal{L} \frac{\mathrm{d} \sigma}{\mathrm{d} \omega} \Delta \omega & \frac{\mathrm{d} \sigma}{\mathrm{d} \omega}=\frac{\mathrm{p}^{2}}{\pi} \frac{\mathrm{d} \sigma}{\mathrm{dt}}=2 \times 10^{-29} \mathrm{~cm}^{2} / \mathrm{sr} \\
& =5 \times 10^{33} \times\left(2 \times 10^{-29}\right) \times 10^{-5} \\
& =1 \text { event } / \mathrm{sec} .
\end{array}
$$

At $10^{-11} \mathrm{~mm}$ pressure, beam-gas interactions would be $\sim 1$ collision/sec. The probability that secondaries from such an interaction reach PCl and PC4 simultaneously is $\sim 10^{-15}$.

For $t=25 \mathrm{Gev}^{2}\left(P_{\perp}=5\right), \mathrm{d} \sigma^{\prime} \mathrm{d} \omega$ would be $6 \times 10^{-34} \mathrm{~cm}^{2} / \mathrm{sr}$ and $\Delta \omega=4 \times 10^{-5} \mathrm{sr}$ could be achieved. Then

$$
N_{P P}=5 \times 10^{33} \times 6 \times 10^{-34} \times 4 \times 10^{-5}=0.43 \text { events } / \mathrm{h} .
$$




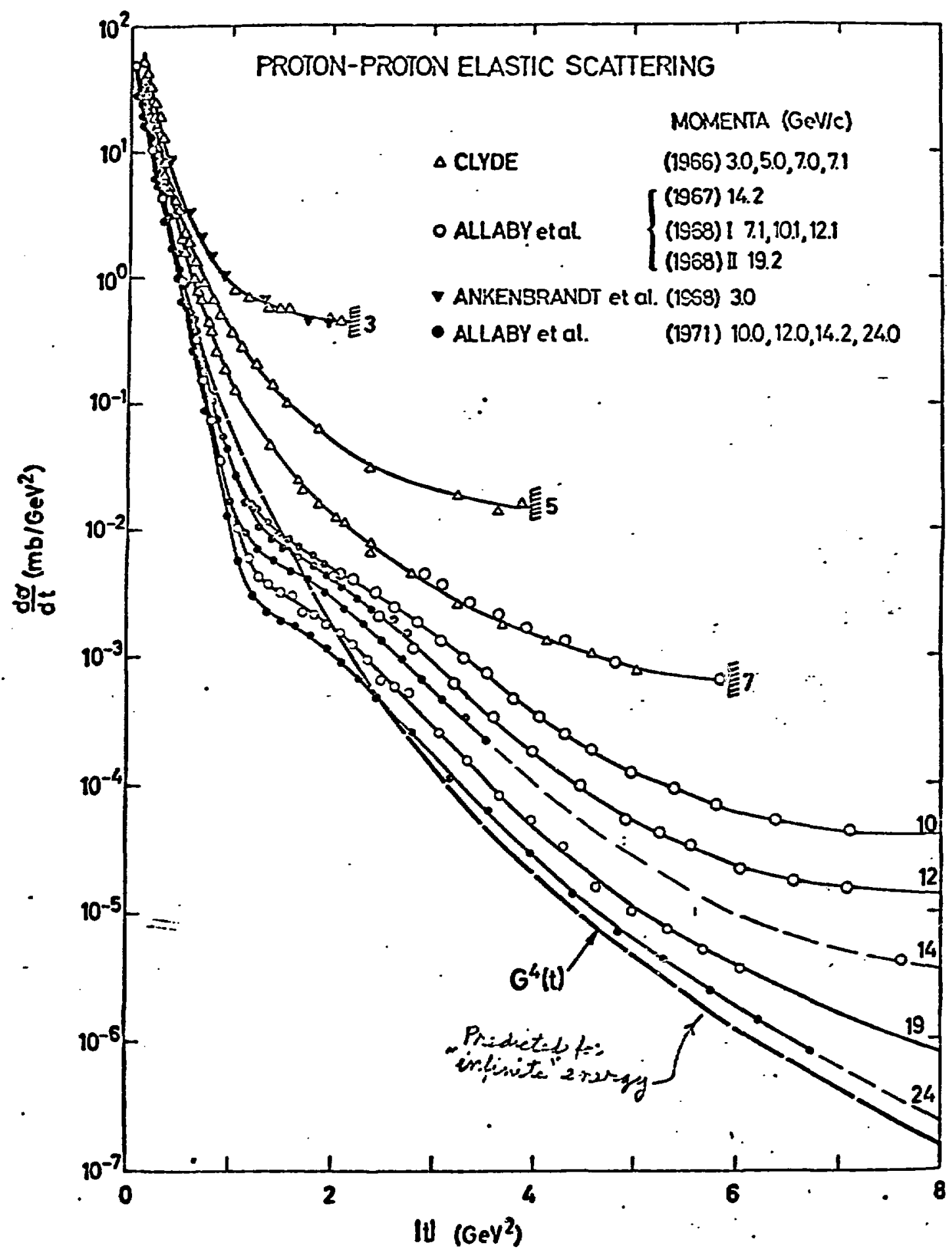

Fig. 1 - 


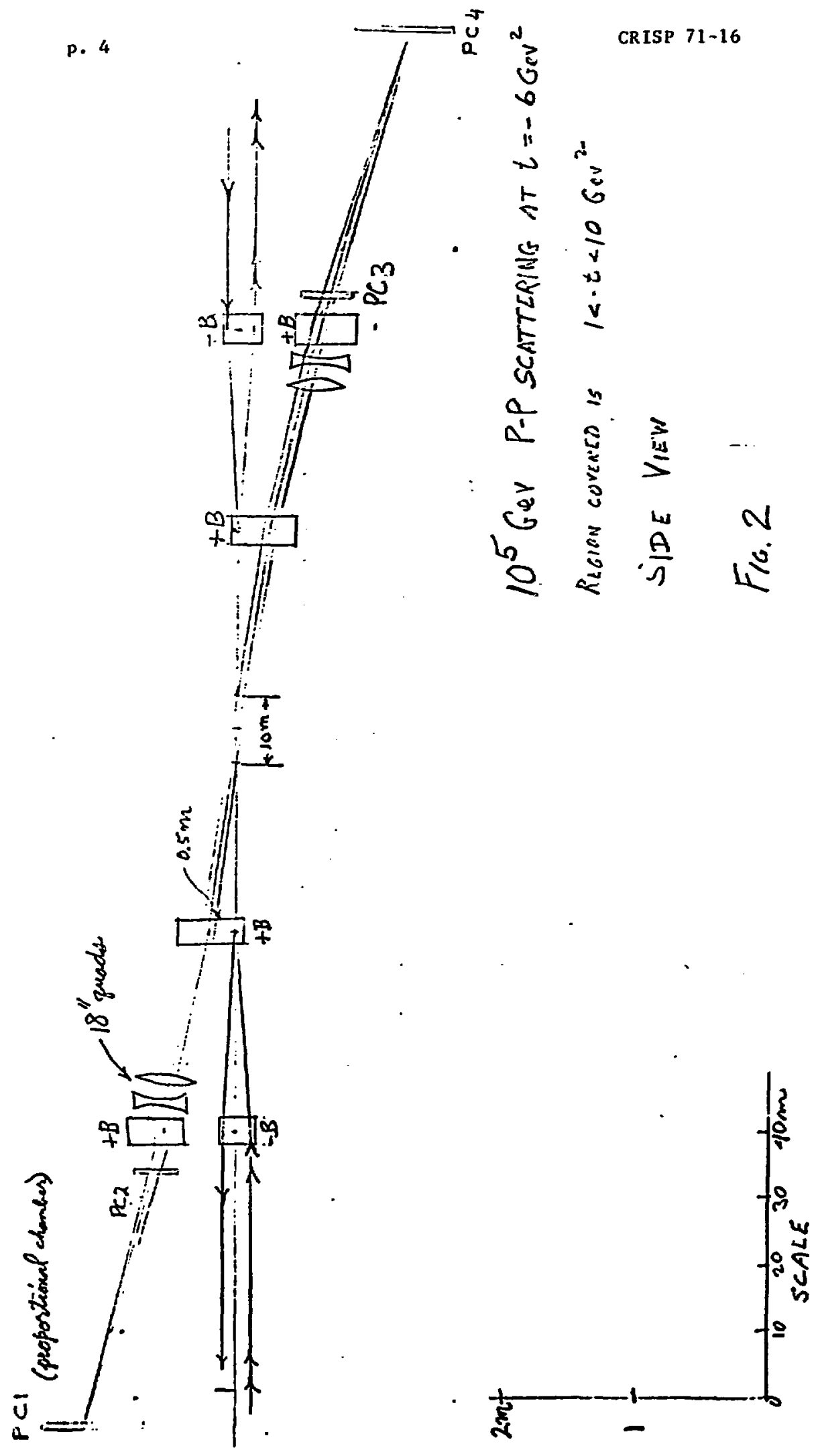

